\title{
Obtención de valores unitarios mediante el método de árboles-tipo para la tasación del arbolado urbano: aplicación en Santiago del Estero (Argentina) y Madrid (España)
}

\author{
Obtaining unit values through the type-trees method for urban trees appraisal: \\ application in Santiago del Estero (Argentina) and Madrid (Spain)
}

\author{
Esperanza Ayuga-Téllez ${ }^{\text {a* }}$, M Lucrecia Contato-Carol b ${ }^{\text {, Claudia García-Ventura a,c }}$ \\ Álvaro Sánchez de Medina a ${ }^{a}$ M Ángeles Grande-Ortiz ${ }^{\text {c }}$, Concepción González-García ${ }^{\mathrm{c}}$

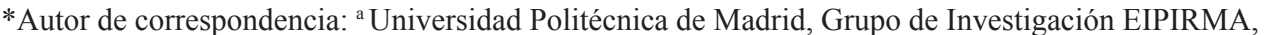 \\ Madrid, España, tel.: +34-913366401, esperanza.ayuga@upm.es \\ ${ }^{\mathrm{b}}$ Universidad Nacional de Santiago del Estero, Facultad de Ciencias Forestales, Santiago del Estero, Argentina. \\ ${ }^{c}$ Universidad Politécnica de Madrid, Grupo de Investigación Tecnologías y Métodos para la Gestión Sostenible, \\ ETS Ingenieros de Montes, Madrid, España.
}

\begin{abstract}
SUMMARY
This work presents a tool for assessing urban trees which allows getting two basic requirements in public management: reduction of costs and simplicity. For this purpose, a methodology based on a multivariate statistical analysis is presented. It allows establishing type-trees in urban trees areas representing and grouping homogenous species and dasometric characteristics simultaneously. Field work was carried out in two different locations: the city of Santiago del Estero (Argentina) and the Arboretum of Madrid Forestry Engineering School (Spain). The methodology groups the trees together in tree groups through the discriminant analysis technique. For each group, the most frequent species in Santiago del Estero were: Citrus auriantum, Tabebuia impetiginosa and Tipuana tipu. In Madrid they were the Ligustrum japonicum, Platanus $x$ hybrida and Populus alba var. bolleana. One type-tree per group was determined, which was representative of all urban trees of that group. The economic value of each type-tree was calculated for each area by the capitalization method. Their unit value is the appraised value of the specimen per unit size. The price per unit of size can be applied to any urban tree in its group, allowing a quick calculation of its basic economic value, low cost and applicable to most valuation formulas. The methodology described allows simplifying the calculation of the economic value of urban trees located in different locations.
\end{abstract}

Key words: tree appraisal; discriminant analysis; municipal management; urban silviculture.

\section{RESUMEN}

Este trabajo ofrece una herramienta para valorar el arbolado urbano, cumpliendo dos requisitos básicos de gestión pública: reducción de costes y sencillez de aplicación. Para ello se presenta una metodología basada en técnicas de análisis estadístico multivariante que permite determinar árboles-tipo en zonas con arbolado urbano que representen y agrupen simultáneamente especies y características dasométricas homogéneas. Se han analizado dos poblaciones diferentes: el centro urbano de Santiago del Estero (Argentina) y el Arboreto de la Escuela Técnica Superior de Ingenieros de Montes, de la Universidad Politécnica de Madrid, campus Moncloa (España). La metodología clasificó de forma automática los árboles en tres grupos mediante análisis discriminante. En cada grupo se selecciona la especie más frecuente, que para el caso de Santiago del Estero, fueron Citrus auriantum, Tabebuia impetiginosa y Tipuana tipu, mientras que en Madrid fueron el Ligustrum japonicum, Platanus x hybrida y Populus alba var. bolleana. Se determinaron los árbolestipo, uno de cada grupo, para cada espacio verde, representativos del conjunto de árboles urbanos. Se calculó el valor económico de los tres árboles tipo, para cada zona, mediante el método de capitalización. Su valor unitario corresponde al valor de tasación de dicho ejemplar por unidad de tamaño. El precio por unidad de tamaño se puede aplicar a cualquier árbol urbano de su mismo grupo, permitiendo un cálculo rápido de su valor económico básico, con bajo coste y aplicable a la mayoría de fórmulas de valoración. La metodología descrita permite simplificar el cálculo del valor económico del arbolado urbano situado en distintas localizaciones.

Palabras clave: valoración del árbol, análisis discriminante, gestión municipal, silvicultura urbana.

\section{INTRODUCCIÓN}

Por lo general, se identifica como arbolado urbano a zonas verdes ubicadas en diferentes lugares, como cinturo- nes verdes, líneas de árboles en aceras, parques, árboles en agrupación dentro de las ciudades o en áreas industriales y otros espacios urbanos (Wenger 1984). La importancia del arbolado urbano y sus beneficios para el hombre y el 
medio ambiente son objetos de estudio desde la década de 1980 (Grande-Ortíz et al. 2012).

La gestión pública de zonas verdes arboladas tiene cada vez una mayor importancia económica y social que no se corresponde con el gasto presupuestario destinado a dicho fin. Por poner un ejemplo, el presupuesto destinado en la Comunidad de Madrid a Medio Ambiente es de tan sólo un $0,007 \%$ del total del gasto. Del total de este porcentaje, el destinado específicamente a Zonas Verdes Urbanas es del 0,16\% (en total, dos millones de euros) (CAM 2014).

Por otro lado, en la Carta de Servicios de Parques y Jardines del Ayuntamiento de Madrid se dice que "los parques, jardines y en definitiva, los espacios públicos urbanos, son elementos imprescindibles en el paisaje y la vida de las ciudades, y como tales debe apreciarse su valor" (Ayuntamiento de Madrid 2013).

En la provincia de Santiago del Estero, con un presupuesto un $40 \%$ inferior al de la Comunidad de Madrid, no existe una línea específica de gasto destinada a zonas verdes urbanas (MSE 2014).

La valoración del arbolado urbano es imprecisa, ya que depende del objetivo de la valoración y de la experiencia del evaluador. Existen varios estudios relacionados con la determinación de un valor monetario del arbolado urbano, ya sea usando fórmulas u otras metodologías como valoración contingente, precios hedónicos y costo de viaje (Contato-Carol et al. 2008, Majumdar et al. 2011, PonceDonoso et al. 2012).

El uso más frecuente de métodos de tasación para el arbolado urbano es consecuencia de las reclamaciones por daños (Jim 2003), litigios judiciales (Schmied y Pillmann 2003) y determinación del patrimonio (Pothier y Millward 2013), por lo cual definir su objetivo es relevante. En la mayoría de los métodos utilizados, el valor se define monetariamente y la valoración pasa por establecer un criterio mensurable y objetivo, considerando aspectos (especie, salud, localización, etc.) o variables diversas (Grande-Ortíz et al. 2012).

Según Ponce-Donoso et al. (2012), se emplean una o más fórmulas de valoración económica del arbolado urbano en Argentina, Australia, Chile, Estados Unidos, España, Finlandia, Francia, Gran Bretaña, Nueva Zelanda, entre otros países, de distinta composición y objetivos de aplicación. También resaltan la necesidad de realizar trabajos de comparación entre metodologías de valoración.

Muchas de las fórmulas descritas en los trabajos de Grande-Ortíz et al. (2012) y de Ponce-Donoso et al. (2012) son fórmulas de valor económico resultado de multiplicar un "valor base" (VB) por factores relacionados con características intrínsecas o extrínsecas del árbol. Los siguientes métodos emplean valores base como producto de "valores económicos unitarios" (VU) por diversas variables dasométricas, medidas en cada ejemplar: el del Council of Tree and Landscape Appraisers (CTLA 2000), con VU por centímetro cuadrado de la sección transversal del tronco; el método Burnley (Moor 1991), con VU por metro cúbico de volumen del árbol (considerado como un cono invertido); la Norma Granada (AEPJP 2007), con VU por centímetro de perímetro del árbol medida a un metro de altura o el método Contato (Contato-Carol 2004), con VU por centímetro cuadrado de la proyección horizontal de la superficie de copa (medida como el área de la elipse formada con ejes perpendiculares, siendo la dirección del eje mayor la del diámetro de mayor proyección de la sombra).

En Argentina, la valoración del arbolado urbano ubicado en espacios de uso público, se encuentra ligada a la determinación del daño de los árboles y a la existencia de normativas municipales que incluyen la valoración. Generalmente, se realiza con participación de personal municipal, que tasa el árbol dañado y propone una cuantía para la multa a aplicar por parte del juez (Cullen 2005). En la mayoría de ciudades europeas, incluida Madrid, la valoración del arbolado urbano está más ligada al patrimonio municipal, como a los costes y beneficios que proporciona al conjunto de ciudadanos (Rotherham 2010) o al incremento del valor del paisaje aportado por el árbol (Notaro y De Salvo 2010).

La dificultad de calcular un valor monetario medio por especie o más aún, obtenerlo para cada individuo (Contato-Carol et al. 2008), aconsejarían una simplificación que permita determinar un valor monetario medio por unidad dasométrica de cada árbol.

Este trabajo tiene como objetivo proporcionar una metodología estadística que permita obtener valores unitarios de las variables dasométricas más utilizadas en las fórmulas de valoración del arbolado urbano (sección transversal del tronco, volumen del árbol, perímetro del tronco y proyección horizontal de la superficie de copa) y comparar los resultados obtenidos de su aplicación en dos espacios verdes de distinta composición florística, y cuyo arbolado se encuentre ubicado en lugares con condiciones diferentes.

\section{MÉTODOS}

Lugares de muestreo. Para el estudio realizado en este trabajo se emplearon datos de dos localizaciones diferentes pero con condiciones climáticas similares: por un lado, el centro de la ciudad de Santiago del Estero (Argentina) cuyos datos pueden consultarse en Contato-Carol (2004), y el Arboreto de la Escuela Técnica Superior de Ingenieros de Montes (ETSIM), sito en el "Campus de Excelencia Internacional" de la ciudad de Madrid, cuyo inventario puede consultarse en Pérez-Fernández (2013). Con ello, se pretenden evaluar las similitudes y diferencias entre ambas ubicaciones a la hora de aplicar la metodología ya que representan dos situaciones diferentes del arbolado urbano: arbolado integrado en el centro de la ciudad principalmente en calles y plazas, y un arboreto dedicado a la docencia.

La ciudad de Santiago del Estero es la capital de la provincia del mismo nombre, situada al noroeste de la República Argentina. La Municipalidad de Santiago del Estero debe gestionar toda la superficie verde de la ciudad y, además, imponer sanciones cuando se producen daños en el 
arbolado, obligando a la dirección gestora del arbolado de la ciudad a instituir un baremo de cuantificación de daños, destrucciones y, en general, contravenciones a la normativa legal. Las sanciones aplicadas por la autoridad municipal ante infracciones sobre el arbolado, se cuantifican basándose en valores económicos unitarios de árboles-tipo del método Contato-Carol (2004).

Santiago del Estero posee un clima semiárido y mesotérmico. El incremento de temperatura que normalmente se produce en las ciudades por efecto de la absorción de calor de edificios y pavimentos, en Santiago del Estero adquiere características notables. La composición florística de la ciudad es de origen diverso y presenta una gran variabilidad de especies arbóreas, arbustivas y herbáceas. Se han detectado 226 especies vegetales diferentes, de las cuales, 132 son arbóreas, 73 arbustos erectos y 21 arbustos trepadores. La superficie total de áreas verdes es de 630 ha. La cubierta arbórea es de 229,25 ha; expresada en $\mathrm{m}^{2} /$ habitante y por barrio, varía entre $54,6 \mathrm{~m}^{2} /$ habitante en los barrios más arbolados y $3,1 \mathrm{~m}^{2} /$ habitante en los menos arbolados (Contato-Carol 2004).

La ciudad de Madrid es la capital de la Comunidad Autónoma del mismo nombre, situada en el centro de España, siendo además la capital de la Nación. El Ayuntamiento de Madrid es el encargado de la tutela y protección del arbolado urbano existente en sus municipios, así como la puesta en práctica de medidas que aseguren su fomento y mejora. Las sanciones incluyen multas y reparación del daño mediante sustitución de los ejemplares afectados por otros similares en especie y edad según la Ley 8/2005, de 26 de diciembre, de Protección y Fomento del Arbolado Urbano de la Comunidad de Madrid (BOCM 2006).

El clima del Arboreto de la ETSIM corresponde también al tipo semiseco y mesotérmico. En el arboreto, se encuentran 2.978 individuos arbóreos de 129 especies arbóreas diferentes, repartidos en 45 zonas de distinta superficie y composición. El recinto cuenta con una superficie arbórea que alcanza las 7,62 ha. Dado que el número de usuarios de este espacio verde es de 1.260, la cubierta arbórea es de 60,48 $\mathrm{m}^{2}$ por usuario (García-Ventura 2013).

Análisis estadístico de datos. En Santiago del Estero (Argentina) se han empleado 447 ejemplares para la determinación de los árboles-tipo y, en el arboreto de la ETSI de Montes de Madrid, 392 ejemplares.

El muestreo realizado en ambos lugares fue un muestreo estratificado tomando como estratos las especies y afijación proporcional. Para obtener el tamaño de muestra se utilizó la varianza correspondiente a la variable diámetro $(d b h)$ que fue la mayor de las correspondientes a las variables dasométricas calculadas.

Las variables medidas por los autores fueron: altura total $(h)$ en m, obtenida como la distancia entre la base del árbol y el extremo superior de la copa sobre el eje del mismo empleando clinómetro Suunto y Vertex; diámetro del fuste $(d b h)$ en $\mathrm{cm}$, medido con forcípula a 1,30 $\mathrm{m}$ sobre el nivel del suelo; proyección horizontal de la superficie de copa $(s c)$ en $\mathrm{m}^{2}$, medida como la proyección de su superficie sobre el suelo y puesto que la mayoría de las copas proyectan formas irregulares, se tomaron dos medidas, la de mayor longitud y su perpendicular (Thren 1993); la edad del arbolado $(t)$, en años, que se obtuvo mediante el dato directo de los Archivos Municipales de plantación en la ciudad de Santiago del Estero (Contato-Carol 2004) y por estimación mediante fórmulas matemáticas y estadísticas en el arboreto de la ETSIM (García-Ventura 2013).

Para el análisis de los datos se utilizaron los programas Microsoft Excel v.2007 y Statgraphics Centurion V.X64, ambos para Windows.

La metodología se desarrolló en cuatro fases: clasificación de especies, selección de las especies más representadas por grupo, selección del árbol-tipo y valoración económica del árbol-tipo.

Fase 1: clasificación de especies. Se realizó una clasificación inicial de las especies en tres grupos, según los criterios habituales en la gestión municipal, en especies de pequeño, mediano y gran porte, mediante los valores máximos que las variables dasométricas $(h, d b h$ y $s c)$ podían alcanzar en su etapa de madurez, se asignó a cada árbol un grupo, dependiendo de la especie (Contato-Carol et al. 2011, Ayuntamiento de Barcelona 2011).

Se realizó un análisis discriminante para validar y automatizar la clasificación inicial (Fisher 1936) donde la variable de clasificación era el grupo en que se incluía la especie: el grupo 1, de pequeño porte, el grupo 2 de porte mediano y el grupo 3 de gran porte. Las variables originales se normalizaron y estandarizaron y se realizó una selección de variables hacia delante. Se seleccionaron las funciones discriminantes atendiendo al valor de la aproximación de $\chi^{2}$ propuesta por Bartlett (1947). Con los árboles muestreados inicialmente y las variables $h, d b h \mathrm{y}$ $s c$ se determinaron dos funciones discriminantes significativas (nivel de significación del $5 \%$ para $\chi^{2}$ ) para realizar la clasificación. Los valores de las probabilidades a priori se asignaron de forma proporcional al porcentaje presente en la muestra. La valoración del éxito de la clasificación se realizó mediante el porcentaje de árboles correctamente clasificados. Se consideró válido un porcentaje superior a 2/3 de casos correctamente clasificados. Al ser los grupos de tamaño desigual (Hair et al. 1999) se aplicó un criterio de valoración denominado "criterio de oportunidad proporcional” o $C_{p r o}$ (Andrus et al. 1986). Así se consideró válida la clasificación si el porcentaje de casos correctamente clasificados superaba el valor de $C_{p r o}$. El $C_{p r o}$ se calculó con la siguiente ecuación:

$$
C_{\text {pro }}=p_{1}^{2}+p_{2}^{2}+p_{3}^{2}
$$

Donde:

$p_{i}=$ proporción de árboles pertenecientes al grupo $i$, con $i$ $=\{1,2,3\}$ 
Fase 2: selección de las especies más representadas por grupo. Para representar a cada uno de los tres grupos, se seleccionaron las especies más frecuentes en la ciudad. Esta frecuencia se calculó, en el caso de Santiago del Estero, a partir de los datos de archivo del vivero municipal (Contato-Carol 2004); y, en el caso de Madrid, comparando con el inventario de arbolado urbano de la Comunidad de Madrid conocido a través de una comunicación personal de la Consejería de Medio Ambiente en el año 2013. También se comprobó que estas especies reunían los mejores requisitos que pueden esperarse del comportamiento de un árbol en la vía pública (condiciones intrínsecas o propias de la especie) según López-González (2004): facilidad de reproducción, cultivo y mantenimiento, mejores condiciones de adaptabilidad al medio urbano, mayor tolerancia a tensiones y agresiones del medio, mayor resistencia a ataques de insectos, hongos, etc., mejor convivencia con otros elementos urbanos (cañerías, cableados aéreos, etc.). Y, por último, mayor apreciación por su aspecto, floración y sombra.

Para validar las especies elegidas, se emplearon los datos de las medidas dasométricas $d b h, h$ y $s c$, comparándose la mediana de cada variable obtenida para la especie tipo, con la mediana del resto de árboles de la muestra de cada grupo. La hipótesis nula de la prueba es la igualdad de medianas, verificándose, para cada zona verde, con el test de Man-Witney.

Fase 3: selección del árbol-tipo. Se seleccionó un árbol representativo de cada grupo. Para ello se obtuvieron los vectores de medias de las variables dasométricas para los árboles seleccionados. De este modo, el árbol-tipo era el ejemplar más próximo al vector de medias de su grupo. Se utilizó la distancia propuesta por Mahalanobis para determinar la proximidad. Esta distancia es adecuada para medir la proximidad de un individuo al centro del grupo en el caso de análisis multivariante con correlación significativa entre las variables empleadas (Contato-Carol 2004).

Fase 4: valoración económica del árbol-tipo. Se calcularon los valores económicos de los árboles-tipo mediante valores básicos obtenidos con la fórmula de capitalización siguiente (Ayuga-Téllez et al. 2004):

$$
\text { Valor }=\left(\frac{P V+C P}{\alpha}\right)(1+i)^{t}+C M A_{j}(1+i)^{t-j}+C E E_{q}(1+i)^{t-q}[2]
$$

Donde,

$P V=$ precio de vivero.

$C P=$ costos de plantación, incluidos los insumos.

$\alpha=$ probabilidad de arraigue.

$t=$ edad al momento de la valoración.

$C M A=$ costos anuales de mantenimiento durante " $\mathrm{j}$ ” años.

$j=$ número de años sobre los cuales se realizan tareas de mantenimiento a cada uno de los ejemplares plantados.

$C E E=$ costos eventuales extraordinarios.

$q=$ un año determinado.

$i=$ tipo de interés (o tasa de rendimiento media).
La tasa de rendimiento media se eligió considerando el trabajo de Weitzman (2001) en que se mostraba para el futuro próximo (de 6 a 25 años) un valor de $3 \%$, y para el medio (de 26 a 75 años) un valor del $2 \%$. Considerando que los árboles de una ciudad tienen una vida media superior a 25 años, se consideró más adecuado el valor del $2 \%$ para el tipo de interés (Ministerio de Medio Ambiente 2005).

Tradicionalmente, la valoración económica forestal ha utilizado expresiones de capitalización para calcular el valor monetario de los árboles (Petersen y Straka 2011). Estas han sido aceptadas por la mayoría de economistas y juristas para su uso en la valoración de bienes intangibles, al menos desde el punto de vista de la toma de decisiones y la gestión (López-Aguillón y López-García 2013), y ha sido recogida en normativas municipales en Latinoamérica (Contato-Carol et al. 2011, López-Aguillón y LópezGarcía 2013).

A partir de los valores básicos, obtenidos con la ecuación [2], se determinaron los valores unitarios de los ejemplares para las diferentes variables dasométricas empleadas en los métodos de valoración más frecuentes, mediante la relación:

$$
V U=\text { Valor/valor de la Variable }
$$

Donde:

Valor $=$ resultado de ecuación [2]

Variable $=\{h, d b h, s c, t\}$

\section{RESULTADOS}

Fase 1 de la metodología en la ciudad de Santiago del Estero. La clasificación inicial según el porte incluía: en el grupo 1, 17 especies de pequeño porte, que en su estado adulto pueden alcanzar $4 \mathrm{~m}$ de altura, diámetros promedio de copa entre $2,5 \mathrm{~m}$ y $3,5 \mathrm{~m}$ y fustes de diámetros normales comprendidos entre 9,5 cm y 17,5 cm: Sapindus saponaria L., Lagerstroemia indica L., Citrus auriantum L., Ligustrum lucidum Ait., Thevetia peruviana (Pers.) Schum, Senna bicapsularis I. et B., Senna spectabilis (DC.) I. et B., Senna corymbosa I. et B., Manihot flabelifolia Pohl y arbustos modificados: Caesalpinia gilliesii Benth, C. pulcherrima Sw., Hibiscus rosa-sinensis. L., Hibiscus syracus L., Nerium oleander L., Callistemon lanceolatus D.C. El 47,2 \% de los 99 árboles de la muestra correspondía a este grupo.

El grupo 2 estaba formado por 24 especies de porte moderado, con alturas medias comprendidas entre los 7 y $10 \mathrm{~m}$; diámetros promedios de copa entre 3,5 y $8,5 \mathrm{~m}$ y fustes entre 28 y $35 \mathrm{~cm}$ de diámetro normal: Tabebuia impetiginosa Standl., Tabebuia heptaphylla Toledo, Tabebuia lapacho Sandw., Tabebuia nodosa Griseb, Jacaranda mimosifolia D. Don., Brachychiton populneum R. Br., Fraxinus pennsylvanica Marshall, Bahuinia forficata Link., Bahuinia variegata L., Leucaena leucocephala De Wit., Melia azedarach L., Melia azedarach L. forma umbraculifera, Cassia fistula L., Ceratonia siliqua L., Cerci- 
dium praecox Harsms., Erythrina crista-galli L., Erythrina corallodendron L., Erythrina speciosa Andr., Acacia aroma Gill.ap.Hook et Arn., Acacia caven Mol., Prosopis vinalillo Stuck., Prosopis ruscifolia Griseb., Parkinsonia aculeata L., Zizyphus mistol Griseb., Geoffroea decorticans Hook et Arn. El 26,17 \% de los árboles de la muestra correspondía a este grupo.

El grupo 3 lo formaban 25 especies de gran porte, con alturas que podían alcanzar los 12-15 m de altura y copas de diámetros promedio que en ocasiones superaban los $20 \mathrm{~m}$ : Tipuana tipu Benth, Pterogynenitens Tul., Grevillea robusta A. Cunn, Enterolobium contortisiliquum Morong., Casuarina cunninghamiana Miq., Peltophorumdubium (Spreng.) Taub., Ceiba chodattii Ravenna, Ceiba speciosa Ravenna, Prosopis nigra Hieron., Prosopis alba Griseb., Schinus molle L., Schinusareira L., Ulmus pumila L., Populus alba L., Populus deltoides Marsh., Populus x canadensis Moench cv.'I-214', Salix humboldtiana Wild., Salix x argentinensis Rag.et R. Alb, Salix babylonica L., Broussonetia papyrife$r a$ (L.) L'Herit., Ficus benjamina L., Ficus elastica Roxb., Ficus lyrata Warb., Pinus halepensis Mill. El 26,62 \% de los árboles de la muestra correspondía a este grupo.

Con las funciones discriminantes mostradas en las ecuaciones [4] y [5], el porcentaje de árboles correctamente clasificados fue del 75,62\% obteniéndose un valor de $C_{p r o}$ del $36 \%$ (ecuación [1]). Por tanto las funciones discriminantes podían considerarse válidas para clasificar los árboles de la ciudad en los tres grupos mencionados. En este caso no fue necesario normalizar las variables.

$$
\begin{aligned}
& \mathrm{F} 1=-0,37 s c+0,48 d b h+0,82 h \\
& \mathrm{~F} 2=-1,13 s c-0,73 d b h+1,37 h
\end{aligned}
$$

Fase 1 de la metodología en el arboreto de la ETSIM de Madrid. La clasificación inicial según el porte incluía: en el grupo 1, 44 especies de árboles de porte pequeño con diámetro de copa inferior a $4 \mathrm{~m}$ y altura menor de 6 m: Abies alba Mill., Abies concolor (Gordon) Lindley ex, Abies masjoanis Masf, Abies nordmanniana (Steven) Spach, Acacia melanoxylon R.Br., Acer campestre L., Acer japonicum Thunb., Acer saccharum Marshall, Arbutus unedo L., Betula pendula Roth, Carpinus betulus L., Castanea sativa Mill., Catalpa bignonioides Walter, Celtis australis L., Cercis siliquastrum L., Calocedrus decurrens (Torr.) Florin, Chamaerops humilis L., Corylus avellana L., Crataegus monogyna Jacq., Eriobotrya japonica (Thunb.) Lindl, Gleditsia triacanthos L., Ilex aquifolium L., Juglans regia L., Juniperus oxycedrus L., Ligustrum japonicum Thunb., Koelreuteria paniculata Laxm, Liquidambar styraciflua L., Malus domestica Borkh., Morus nigra L., Olea europaea var. europaea L., Pinus radiata D.Don, Pinus uncinata Raymond, Pistacia vera L., Pittosporum tobira (Thunb.) W.T.Aiton, Platycladus orientalis (L.) Franco, Prunus dulcis (Mill.) D.A. Webb, Prunus laurocerasus L., Prunus padus L., Pseudotsuga menzie- sii (Mirb.) Franco, Punica granatum L., Quercus ilex ssp. ballota L., Quercus robur L., Sorbus domestica L., Tilia cordata Mill., Tilia platyphyllos Scop. El 24,49 \% de los árboles de la muestra correspondían a este grupo.

En el grupo 2 se incluían 22 especies de porte mediano con diámetro de copa entre 4 y 6 m y altura entre 6 y $15 \mathrm{~m}$ : Acer negundo L., Aesculus hippocastanum L., Ailanthus altissima (Mill.) Swingle, Cupressus arizonica Greene, Fraxinus angustifolia Vahl, Fraxinus excelsior L., Larix decidua Mill., Magnolia grandiflora L., Melia azedarach L., Pinus wallichiana A.B.Jacks., Pinus halepensis Mill., Pinus nigra J.F.Arnold, Pinus pinaster Ait., Pinus pinea L., Platanus $x$ hybrida (Mill.) Münchh., Quercus canariensis Willd., Quercus suber L., Robinia pseudoacacia L., Sophora japonica (L.) Schott, Tetraclinis articulata (Vahl) Masters, Ulmus pumila L., Washingtonia robusta H.Wendl. El 47,7 \% de los árboles de la muestra correspondían a este grupo.

En el grupo 3, 13 especies de porte grande, con diámetro de copa mayor de $6 \mathrm{~m}$ y altura superior a $15 \mathrm{~m}$ : Abies pinsapo Boiss., Cedrus atlantica (Endl.) Hook.f., Cedrus deodara (Roxb.) G.Don, Cupressus sempervirens L., Eucalyptus camaldulensis Dehnh., Eucalyptus globulus Labill., Pinus canariensis C.Sm. ex DC., Pinus coulteri D.Don, Populus alba L., Populus alba var. bolleana (Lauche) Otto, Populus simonii Carrière, Sequoia sempervirens Endl., Sequoiadendron giganteum (Lindl.) J. Buchholz. El $27,81 \%$ de los árboles de la muestra pertenecían a este grupo.

Con las funciones discriminantes, mostradas en las ecuaciones [6] y [7], el porcentaje de árboles correctamente clasificados fue del $75 \%$ obteniéndose un valor de $C_{p r o}$ del 36,53 \% (ecuación 1). Por tanto las funciones discriminantes podían considerarse válidas para clasificar los árboles de la ciudad en los tres grupos mencionados.

$$
\begin{aligned}
& \mathrm{F} 1=-0,70 \operatorname{Ln}(s c)^{1 / 2}+0,48 \operatorname{Ln}(d b h)+0,95(h)^{1 / 2} \\
& F 2=1,10 \operatorname{Ln}(s c)^{1 / 2}+0,05 \operatorname{Ln}(d b h)-0,26(h)^{1 / 2}
\end{aligned}
$$

Donde las variables se normalizaron mediante transformación logarítmica, y/o raíz cuadrada

Fase 2 de la metodología en la ciudad de Santiago del Estero. Las especies más frecuentes en la ciudad y que cumplían con las características descritas en la metodología fueron el naranjo agrio (Citrus auriantum) en el grupo 1 , con un $15 \%$ de presencia, el lapacho (Tabebuia impetiginosa Standl.) en el grupo 2, con 12,89\% y la tipa blanca (Tipuana tipu) en el grupo 3, con 3,79\%.

Mediante la prueba de Man-Witney para comprobar la hipótesis de la igualdad de la mediana entre la de la especie seleccionada y el resto de árboles por grupo, con los datos de los 1.078 árboles seleccionados en la ciudad, y un $95 \%$ de confianza, no se pudo rechazar la hipótesis de igualdad de las medianas en la mayoría de los casos (cuadro 1). 
Cuadro 1. Resultados del test de Man-Witney para comparación de medianas entre especie-tipo y grupo representado por dicha especie ( $P$-valores).

Results of Man-Whitney test for comparison of medians between type species and group represented by the species $(P$-values).

\begin{tabular}{ccccccc}
\hline Lugar & \multicolumn{3}{c}{ Santiago del Estero } & \multicolumn{3}{c}{ Arboreto de la ETSIM } \\
\hline \multirow{2}{*}{ Variable } & Grupo 1 & Grupo 2 & Grupo 3 & Grupo 1 & Grupo 2 & Grupo 3 \\
& $n=83$ & $n=68$ & $n=42$ & $n=3$ & $n=22$ & $n=24$ \\
\hline$d b h$ & 0,4438 & 0,0023 & 0,0599 & 0,6731 & 0,1387 & 0,0015 \\
$h$ & 0,7698 & 0,0371 & 0,9564 & 0,9194 & 0,0526 & 0,1360 \\
$s c$ & 0,6445 & 0,0877 & 0,9326 & 0,5151 & 0,0580 & 0,0999 \\
\hline
\end{tabular}

$n$ : número de ejemplares de la especie seleccionada incluidos en la muestra, para obtener el árbol-tipo; dbh: diámetro del fuste, medido a 1,30 m del suelo $(\mathrm{cm}) ; h$ : distancia entre la base del tronco y el extremo superior de la copa del mismo, medido sobre su eje (m), sc: superficie de copa medida como el área del círculo de diámetro equivalente al ancho de copa $\left(\mathrm{m}^{2}\right)$.

Fase 2 de la metodología en el arboreto de la ETSIM de Madrid. Las especies más frecuentes en la ciudad de Madrid, según la comunicación personal transmitida por la Consejería de Medio Ambiente de la Comunidad de Madrid a los autores de este trabajo, que cumplían con las características descritas en la metodología fueron: el aligustre del Japón (Ligustrum japonicum) en el grupo 1, con un 4,2 \% de presencia; el plátano de sombra (Platanus $x$ hybrida) en el grupo 2 con 11,8 \%; y el álamo plateado (Populus alba var. bolleana) en el grupo 3, con 3,79\%.

No se pudo rechazar la hipótesis de igualdad de las medianas en la mayoría de las variables consideradas, entre los árboles de la especie seleccionada y el resto de árboles por grupo con un $95 \%$ de confianza (cuadro 1).

Fase 3 de la metodología en ambos lugares de aplicación. En los árboles de las especies seleccionadas la edad media aumentó con el porte, en ambas localidades. En el arboreto de la ETSIM se observaron valores medios para cada grupo superiores a los valores medios obtenidos en los grupos de la ciudad de Santiago del Estero (cuadro 2).

Para la ciudad de Santiago del Estero, las distancias de Mahalanobis entre los árboles-tipo y el vector de medias fueron: 0,17 en el grupo 1; 0,25 en el grupo 2 y 0,59 en el grupo 3. Para el arboreto de la ETSIM, las distancias de Mahalanobis entre los árboles-tipo y el vector de medias fueron: 0,3 en el grupo 1; 0,65 en el grupo 2 y 0,4 en el grupo 3. Los arboles tipo seleccionados con la mínima distancia de Mahalanobis (cuadro 3) mostraron características similares por grupo a los valores medios (cuadro 3 ).

Fase 4 de la metodología en ambos lugares de aplicación. Los valores básicos calculados con la ecuación 2 para Santiago del Estero fueron menores que en el arboreto (cuadro 4) y se observó que, en general, los costes fueron inferiores para la primera ciudad, excepto en el caso de los costes extraordinarios que están incluidos en el presupuesto general de gestión del arboreto.

Los valores unitarios, obtenidos mediante la ecuación 3 , reflejan la diferencia de costes entre ambas localizacio- nes. Entre el grupo 1 y el grupo 2 de la misma localización, los valores unitarios fueron similares, mientras que los del grupo 3 fueron mayores. En las dos ubicaciones, el valor unitario por superficie de copa del grupo 1 fue superior a la del grupo 2, incluso en Santiago del Estero fue superior a la del grupo 3 (cuadro 5).

\section{DISCUSIÓN Y CONCLUSIONES}

Este trabajo ofrece una herramienta que consigue valorar el arbolado urbano cumpliendo dos requisitos básicos de gestión pública: a) reducción de costes (los árboles-tipo permiten reducir el cálculo del valor económico de todos los árboles de la ciudad a sólo tres ejemplares) y b) sencillez de aplicación (la clasificación puede automatizarse, basándose en las tres funciones de clasificación obtenidas en con el análisis discriminante).

Los resultados de las pruebas y medidas de éxito de la clasificación muestran cómo, con este método, la agrupación de especies según el porte es válida y los resultados para diferentes lugares son similares en cuanto a capacidad de agrupar de forma homogénea los árboles de ciudad. Por ello, con las funciones discriminantes calculadas para los árboles del lugar considerado, se puede automatizar la inclusión de una nueva especie en cualquiera de los tres grupos. En todo caso, es posible mejorar la metodología analizando algunos resultados. Las especies-tipo seleccionadas son adecuadas para la mayoría de los grupos formados excepto para el grupo 2 de Santiago del Estero donde 2 de los 3 valores $P$ calculados al $95 \%$ de confianza no permiten aceptar la hipótesis de que no hay diferencias entre las medianas de las variables $(d b h$ y $h)$ de la especie tipo y del resto de las especies del grupo tal como se muestra en el cuadro 1. Posiblemente la gran diversidad de especies de este grupo en la zona sugiere mejorar la definición del grupo y, por tanto, de las especies que lo forman, consiguiendo más homogeneidad con la especie tipo. Además, la verificación de la hipótesis de igualdad de medianas es más alta en el arboreto (se verifica en un $78 \%$ de los casos) que en Santiago del Estero (se verifica en un $66 \%$ ); esto 
Cuadro 2. Vectores de medias para las especies-tipo seleccionadas.

Mean vectors for species-type selected.

\begin{tabular}{ccccccc}
\hline & \multicolumn{3}{c}{ Santiago del Estero } & \multicolumn{3}{c}{ Arboreto de la ETSIM } \\
\cline { 2 - 7 } Variable & Grupo 1 & Grupo 2 & Grupo 3 & Grupo 1 & Grupo 2 & Grupo 3 \\
& $n=83$ & $n=68$ & $n=42$ & $n=3$ & $n=22$ & $n=24$ \\
\hline$c$ & 20,13 & 63,52 & 110,21 & 52,57 & 84,65 & 144,50 \\
$d b h$ & 6,41 & 20,22 & 35,08 & 16,73 & 26,95 & 46,00 \\
$h$ & 2,71 & 5,28 & 8,08 & 5,17 & 11,80 & 17,33 \\
$s c$ & 3,85 & 17,24 & 47,63 & 11,33 & 29,78 & 12,85 \\
$t$ & 15 & 43 & 79 & 39 & 64 & 109 \\
\hline
\end{tabular}

$n$ : número de ejemplares de la especie seleccionada incluidos en la muestra, para obtener el árbol-tipo; $c$ : perímetro normal o longitud del perímetro del fuste, perpendicular al eje del mismo y tomado a $1,30 \mathrm{~m}$ sobre el nivel del suelo $(\mathrm{cm}) ; d b h$ : diámetro del fuste, medido a $1,30 \mathrm{~m}$ del suelo $(\mathrm{cm}) ; h$ : distancia entre la base del tronco y el extremo superior de la copa del mismo, medido sobre su eje (m); sc: superficie de copa medida como el área del círculo de diámetro equivalente al ancho de copa $\left(\mathrm{m}^{2}\right)$; $t$ :edad medida como el número de años transcurridos desde la germinación de la semilla estimada para cada ejemplar.

Cuadro 3. Características del árbol-tipo de cada grupo para ambos lugares de muestreo.

Features of the type-trees of each group in both sample locations.

\begin{tabular}{lccccccc}
\hline Lugar & \multicolumn{3}{c}{ Santiago del Estero } & \multicolumn{3}{c}{ Arboreto de la ETSIM } \\
\hline Grupo & & Grupo 1 & Grupo 2 & Grupo 3 & Grupo 1 & Grupo 2 & Grupo 3 \\
\hline \multirow{4}{*}{ Variable } & $c$ & 16,0 & 74,0 & 142,0 & 32,0 & 93,9 & 166,2 \\
& $d b h$ & 5,9 & 23,5 & 45,2 & 10,2 & 29,9 & 52,9 \\
& $g$ & 20,3 & 435,6 & 1604,6 & 81,7 & 702,1 & 2197,9 \\
& $h$ & 2,5 & 6,0 & 10,0 & 5,0 & 14,0 & 19,0 \\
& $d c$ & 1,2 & 5,0 & 9,5 & 2,5 & 7,3 & 4,5 \\
& $s c$ & 1,1 & 19,6 & 70,9 & 4,7 & 41,8 & 15,7 \\
& $t$ & 12 & 54 & 101 & 24 & 71 & 125 \\
\hline
\end{tabular}

$c$ : perímetro o longitud del perímetro del fuste, perpendicular al eje del mismo y tomado a 1,30 m sobre el nivel del suelo (cm); $d b h$ : diámetro del fuste, medido a 1,30 m del suelo $(\mathrm{cm}) ; g$ : superficie de la intersección del fuste con un plano perpendicular a su eje longitudinal, tomado a 1,30 m sobre el nivel del suelo $\left(\mathrm{cm}^{2}\right) ; h$ : distancia entre la base del tronco y el extremo superior de la copa del mismo, medido sobre su eje (m); $d c$ : ancho de la copa, medido a través de la proyección de los dos extremos de la misma sobre el terreno; como las copas proyectan formas irregulares, se siguió el criterio de tomar el eje mayor y el perpendicular a éste. Así, el valor promedio se obtiene como media aritmética (m); sc: superficie de copa medida como el área del círculo de diámetro equivalente al ancho de copa $\left(\mathrm{m}^{2}\right)$; $t$ : edad medida mediante el número de años transcurridos desde la germinación de la semilla estimada para cada ejemplar.

Cuadro 4. Valores básicos $(€)$ de los arboles-tipo obtenidos con la fórmula de capitalización.

Basic values $(€)$ obtained through capitalization formula for each group in both sample locations.

\begin{tabular}{clcccccc}
\hline Lugar & & \multicolumn{3}{c}{ Santiago del Estero } & \multicolumn{3}{c}{ Arboreto de la ETSIM } \\
\hline Grupo & & Grupo 1 & Grupo 2 & Grupo 3 & Grupo 1 & Grupo 2 & Grupo 3 \\
\hline \multirow{3}{*}{ Variable } & $P V$ & 1,53 & 2,04 & 2,04 & 29,83 & 12,72 & 13,06 \\
& $C P$ & 5,10 & 5,10 & 5,10 & 360,00 & 420,00 & 420,00 \\
& $C E E$ & 1,79 & 1,79 & 1,79 & 5,20 & 5,20 & 5,20 \\
& $\alpha$ & 0 & 77,61 & 196,86 & 0 & 0 & 0 \\
Valoración & Curos) & 0,75 & 0,75 & 0,75 & 0,75 & 0,75 & 0,75 \\
\hline
\end{tabular}

$P V$ : precio de la planta en vivero $(€)$; $C P$ : costes de plantación, incluidos insumos $(€)$; $C M A$ : costos de mantenimiento anual sabiendo que en ambos casos se realizaron trabajos de mantenimiento a lo largo de 5 años después de la plantación ( $€ /$ año); $C E E$ : costos eventuales extraordinarios (en dos árboles-tipo se realizaron dos intervenciones extraordinarias a los 15 y 20 años de edad, cada una de ellas con costes de 12,75€ y 25,51€, respectivamente); $\alpha$ : probabilidad de arraigo de la especie; $t$ :edad medida mediante el número de años transcurridos desde la germinación de la semilla estimada para cada ejemplar. 
Cuadro 5. Valores unitarios por grupo y lugar, según diferentes variables dasométricas.

Unitary values according to different dasometric variables for each group in both sample locations.

\begin{tabular}{cccccccr}
\hline Lugar & \multicolumn{3}{c}{ Santiago del Estero } & \multicolumn{3}{c}{ Arboreto de la ETSIM } \\
\hline Grupo & & Grupo 1 & Grupo 2 & Grupo 3 & Grupo 1 & Grupo 2 & Grupo 3 \\
\hline & $c$ & 1,4 & 1,8 & 2,3 & 27,4 & 26,1 & 43,0 \\
& $d$ & 3,7 & 5,5 & 7,3 & 85,8 & 82,1 & 135,2 \\
Variable & $g$ & 1,1 & 0,3 & 0,2 & 10,7 & 3,5 & 3,3 \\
& $h$ & 8,8 & 21,7 & 33,0 & 175,1 & 175,3 & 376,5 \\
& $d c$ & 18,3 & 26,0 & 34,7 & 350,2 & 336,1 & 1589,8 \\
& $s c$ & 19,9 & 6,6 & 4,6 & 186,3 & 58,7 & 455,7 \\
& $t$ & 1,8 & 2,4 & 3,3 & 36,5 & 34,6 & 57,2 \\
\hline
\end{tabular}

$c$ : perímetro del perímetro del fuste, perpendicular al eje del mismo y tomado a $1,30 \mathrm{~m}$ sobre el nivel del suelo (cm); $d b h$ : diámetro del fuste, medido a $1,30 \mathrm{~m}$ del suelo $(\mathrm{cm}) ; \mathrm{g}$ : superficie de la intersección del fuste con un plano perpendicular a su eje longitudinal, tomado a 1,30 $\mathrm{m}$ sobre el nivel del suelo $\left(\mathrm{cm}^{2}\right) ; h$ : distancia entre la base del tronco y el extremo superior de la copa del mismo, medido sobre su eje (m); $d c$ : ancho de la copa, medido a través de la proyección de los dos extremos de la misma sobre el terreno; como las copas proyectan formas irregulares, se siguió el criterio de tomar el eje mayor y el perpendicular a éste. Así, el valor promedio se obtiene como media aritmética (m); sc: superficie de copa medida como el área del círculo de diámetro equivalente al ancho de copa $\left(\mathrm{m}^{2}\right)$; $t$ : edad medida mediante el número de años transcurridos desde la germinación de la semilla estimada para cada ejemplar.

puede reflejar las diferentes condiciones de crecimiento en ambas localizaciones, debido probablemente a los problemas de crecimiento de los árboles en la ciudad (Churkina et al. 2014) y más aún en zonas de climas áridos (Martínez et al. 2014). Por otro lado, el grupo 3 del Arboreto presenta unos resultados que no permiten verificar la igualdad de medianas en cuanto a diámetro entre la especie-tipo ( $\mathrm{PO}$ pulus alba var. bolleana) y el resto del grupo. Esto puede ser debido a la mezcla de especies de crecimiento rápido (como Populus alba var. bolleana, Eucalyptus globulus y Cedrus deodara) con especies de crecimiento más lento como Sequoia sempervirens, Sequoiadendron giganteum o Abies pinsapo. Se trata de especímenes de alturas similares con diámetros diferentes (López-González 2004).

En el cuadro 2 se observa que los vectores de medias de las especies-tipo de los grupos formados en Santiago del Estero son inferiores a los correspondientes al arboreto de la ETSIM, como era de esperar, ya que este último es un espacio verde cuyas condiciones son, en líneas generales, más favorables para el desarrollo del arbolado (Sánchez de Lorenzo-Cáceres 2005). Los ejemplares de la ciudad argentina son árboles de calle en su gran mayoría, mientras que los ejemplares del arboreto se pueden clasificar como elementos de un parque con fines académicos. La aplicación del método de árboles-tipo en diferentes localizaciones urbanas y diferentes ciudades (como cinturones verdes, pequeños, parques infantiles o áreas industriales) permite comprobar si existen diferencias significativas en las dimensiones medias dependiendo de los tipos de espacios verdes.

La correlación entre variables dasométricas mostrada en trabajos anteriores (Ayuga-Téllez et al. 2006, Reque et al. 2007) y el hecho de que el análisis discriminante asigne nuevos individuos a los grupos aconseja el uso de la distancia de Mahalanobis, que ha sido la empleada en este trabajo, para seleccionar los árboles-tipo. Las distancias obtenidas entre valores medios y árboles tipo son todas inferiores a 1 y en el mismo rango de diferencias, lo que permite verificar que los árboles tipo son representativos del grupo. En los trabajos previos de Contato-Carol (2004) y García-Ventura (2013) se emplea el método de árboles-tipo usando la mínima distancia euclídea al vector de medias, con las variables $d b h$ y $h$, para seleccionar los ejemplares. Los resultados de estos trabajos muestran ejemplares de árboles-tipo con valor económico y dimensiones inferiores a los obtenidos en este trabajo.

Se puede concluir que los valores obtenidos en los análisis discriminantes muestran la validez de las funciones discriminantes para clasificar, según el porte, los árboles de ambas ciudades en tres grupos de especies (pequeño, mediano y grande). Los grupos de especies clasificados según el porte se pueden caracterizar adecuadamente mediante una única especie-tipo, seleccionada como la más frecuente de cada grupo presente en las calles de la ciudad y que presenta características apropiadas para el arbolado urbano. De este modo, cada especie seleccionada se describe con un "árbol-tipo" de dimensiones próximas a los valores medios de la población.

Los valores unitarios permiten calcular valores económicos de cualquier ejemplar, empleando como valor básico del ejemplar el producto del valor por unidad dasométrica y la medida sobre el árbol de dicha variable considerada en la fórmula de valoración correspondiente.

Los valores unitarios son muy diferentes según la localización, siendo mucho mayores los obtenidos en el arboreto de la ETSIM. Esto se debe a que en Madrid el valor de las variables que intervienen en el método de capitalización es mayor. El precio de la planta en vivero es casi 10 veces mayor en Madrid que en Santiago del Estero, y los costes de plantación y mantenimiento son casi 80 ve- 
ces más altos en Madrid. De este modo, el valor unitario también permite detectar las diferencias entre los costes de gestión del arbolado.

En resumen, la metodología propuesta presenta resultados similares en cuanto a la capacidad para formar grupos homogéneos de arbolado en dos lugares diferentes, por el espacio urbano que representan, como son el centro de la ciudad de Santiago del Estero y el arboreto de la ETSIM de la ciudad de Madrid. Este hecho sugiere la posibilidad de poder extrapolar esta metodología en próximos estudios a cualquier ciudad y permitir el cálculo del patrimonio municipal o la estimación de daños en el arbolado, por catástrofes naturales, de forma rápida y basada en fórmulas económicas aceptadas por la mayoría de juristas y economistas (Jim 2003).

\section{REFERENCIAS}

AEPJP (Asociación Española de Parques y Jardines Públicos, ES). 2007 Método para la valoración de árboles y arbustos ornamentales: Norma Granada: revisión 1999. Madrid, España. Asociación Española de Parques y Jardines Públicos. 53 p.

Andrus DM, E Silver, DE Johnson. 1986. Status brand management and gift purchase: a discriminant analysis. Journal of Consumer Marketing 3(1): 5-13.

Ayuga-Téllez E, ML Contato-Carol, MA Grande-Ortiz. 2004. Valoración del arbolado urbano de Santiago del Estero (Argentina) mediante métodos paramétricos. Los árboles en el Paisaje Urbano. Madrid, España. Asociación Española de Arboricultura. p. 237-243.

Ayuga-Téllez E, AJ Martin Fernández, C González García, E Martínez Falero. 2006. Estimation of non-parametric regression for dasometric measures. Journal of Applied Statistics 33(8): 819-836.

Ayuntamiento de Barcelona. 2011. Gestión del arbolado viario de Barcelona. Consultado 26 sept. 2014. Disponible en https:// w110.bcn.cat/MediAmbient/Continguts/Vectors_Ambientals/Espais_Verds/Documents/Traduccions/Plangestionarboladoviarioben_cast.pdf

Ayuntamiento de Madrid. 2013. Carta de Servicios de Parques y Jardines. Consultado 26 sept 2014. Disponible en http:// www.madrid.es/

Bartlett M. 1947. Multivariate analysis. Journal of the Royal Statistical Society, Supplement Series B. 9: 176-197.

BOCM (Boletín Oficial de la Comunidad de Madrid, ES). 2006. Ley 8/2005, de 26 de diciembre, de Protección y Fomento del Arbolado Urbano de la Comunidad de Madrid. Consultado 8 abr. 2014. Disponible en http://www.madrid.org/ wleg/servlet/Servidor?cdestado $=$ P\&nmnorma $=3387 \&$ opci on $=$ VerHtml

CAM (Comunidad de Madrid, ES). 2014. Presupuestos Generales. Consultado 26 sept. 2014. Disponible en http://www. madrid.org/

Churkina G, R Grote, TM Butler, M Lawrence. 2015. Natural selection? Picking the right trees for urban greening. Environmental Science \& Policy 47: 12-17.

Contato-Carol ML. 2004. Análisis estadístico multivariante del arbolado urbano de Santiago del Estero (Argentina) y estimación de su valor mediante modelos econométricos. Tesis
Doctoral. Madrid, España. ETSI de Montes. Universidad Politécnica de Madrid. 278 p.

Contato-Carol ML, E Ayuga-Téllez, MA Grande-Ortiz. 2008. A comparative analysis of methods for the valuation of urban trees in Santiago del Estero, Argentina. Spanish Journal of Agricultural Research 6(3): 341-352.

Contato-Carol ML, E Ayuga-Téllez, MA Grande-Ortiz, C González-García. 2011. Obtención de Árboles Tipo para la Gestión del Arbolado Urbano de Santiago del Estero (Argentina). VI Congreso Ibérico de AgroIngeniería 5-7 de julio, Évora, Portugal. Consultado 8 abr. 2014. Disponible en http://oa.upm.es/12942/1/INVE_MEM_2011_108300.pdf

CTLA (Council of Tree and Landscape Appraisers, US). 2000. Guide for plant appraisal. $9^{\text {th }}$ ed. Council of Tree and Landscape Appraisers, ISA. Champaign, Illinois, United States. Savoy. $143 \mathrm{p}$.

Cullen S. 2005. Tree appraisal: chronology of North American industry guidance. Journal of Arboriculture 31(4):157-162.

Fisher RA. 1936. The Use of Multiple Measurements in Taxonomic Problems. Annals of Eugenics 7(2): 179-188.

García-Ventura C. 2013. Comparación de métodos de valoración de arbolado urbano y su aplicación al arboreto de la ETSI de Montes. Trabajo Fin de Grado Ingeniero de Montes. Madrid, España. ETSI de Montes, Universidad Politécnica de Madrid. $256 \mathrm{p}$.

Grande-Ortiz MA, E Ayuga-Téllez, ML Contato-Carol. 2012. Methods of tree appraisal: a review of their feature sand application possibilities. Arboriculture and Urban Forestry 38(4): 130-140.

Hair JF, RD Anderson, RL Tatham, WC Black. 1999. Análisis Multivariante, $5^{\text {a }}$ ed. Madrid, España. Prentice Hall. 832 p.

Jim CY. 2003. Protection of urban trees from trenching damage in compact city environments. Cities 20(2): 87-94

López-Aguillón R, M López-García. 2013. Evaluación y comportamiento paisajístico de especies nativas en Linares, NL, 16 años de evaluación. Revista Mexicana de Ciencias Forestales, 4(17): 164-173.

López González G. 2004. Guía de los árboles y arbustos de la Península Ibérica y Baleares. Madrid, España. Mundi-Prensa. $894 \mathrm{p}$.

Majumdar S, JDeng, Y Zhang, C Pierskalla. 2011. Using contingent valuation to estimate the willingness of tourists to pay for urban forests: A study in Savannah, Georgia. Urban Forestry \& Urban Greening 10(4): 275-280.

Martínez, CF, MA Cantón, FA Roig. 2014. Incidencia del déficit hídrico en el crecimiento de árboles de uso urbano en ciudades de zonas áridas. Caso de Mendoza, Argentina. Interciencia 39(12): 890-897.

Moor GM. 1991. Amenity tree evaluation: A revised method. In The Scientific Management of Plants in the Urban Environment. Proceedings of the Burnley Centenary Conference Centre for Urban Horticulture, Melbourne, Australia. p. 166-171.

MSE (Municipalidad de Santiago del Estero, AR). 2014. Presupuesto. Consultado 26 de sep. 2014. Disponible en:

http://www.meconse.gov.ar/Presupuesto/2014/Mensaje_Ley/ Mensaje_2014.pdf

Ministerio de Medio Ambiente. 2005. Valoración Económica de Espacio Forestal del Territorio Histórico de Guipúzcoa. Servicio de Inventario Forestal. 12 p. Consultado 17 jul. 2015. Disponible en http://www.nasdap.ejgv.euskadi.eus/ 
r5015135/es/contenidos/informacion/if_valor_econom/ es_dapa/adjuntos/valoracion_economica_gipuzkoa.pdf

Notaro S, M de Salvo. 2010. Estimating the economic benefits of the landscape function of ornamental trees in a sub-Mediterranean area. Urban Forestry and Urban Greening 9(2): 71-81.

Petersen K, T Straka. 2011. Specialized discount cash flow analysis formulas for valuation of benefits and cost of urban trees and forest. Arboriculture and Urban Forestry 37: 200-206.

Ponce-Donoso M, Ó Vallejos-Barra, G Daniluk-Mosquera. 2012. Comparación de fórmulas chilenas e internacionales para valorar el arbolado urbano. Bosque 33(1): 69-81.

Pothier AJ, AA Millward. 2013. Valuing trees on city-center institutional land: an opportunity for urban forest management. Journal of Environmental Planning and Management 56(9): 1380-1402.

Reque JA, M Sarasola, J Gyenge, ME Fernández. 2007. Caracterización silvícola de ñirantales del norte de la Patagonia para la gestión forestal sostenible. Bosque 28(1): 33-45.

Rotherham ID. 2010. Thoughts on the politics and economics of urban street trees. Arboricultural Journal 33(2): 69-75. Sánchez de Lorenzo-Cáceres JM. 2005. Diversidad del arbolado viario mediterráneo. XXXII Congreso PARJAP de la Asociación Española de Parques y Jardines Públicos. Almería. Consultado 7 jul. 2015. Disponible en http://www.arbolesornamentales.es

Schmied A, W Pillmann. 2003. Tree protection legislation in European cities. Urban Forestry and Urban Greening 2 (2): $115-124$

Thren M. 1993. Dasometría. Serie técnica Forestal. Vol. 1. Santiago del Estero, Argentina. Proyecto GTZ/UNSE, Facultad de Cs. Forestales, UNSE. 250 p.

Titus K, JA Mosher, BK Williams. 1984. Chance-corrected Classification for Use in Discriminant Analysis: Ecological Applications. American Midland Naturalis 111(1): 1-7.

Weitzman ML. 2001. Gamma Discounting. The American Economic Review 91(1): 260-271.

Wenger K. 1984. Urban Forestry. In Wenger K ed. Forestry Handbook. Washington, United States. Wiley Interscience. p. 887-983.

Recibido: 03.11.14

Aceptado: 16.10 .15 